\title{
On the role of the $\mathrm{II}(1 / 2 \mathrm{~g})$ state in spontaneous dissociation of krypton and xenon dimer ions
}

\author{
J. Fedor ${ }^{\text {a }}$, O. Echt ${ }^{\text {b,*}}$, K. Głuch ${ }^{\text {c }}$, S. Matt-Leubner ${ }^{\text {e }}$, P. Scheier ${ }^{\text {e }}$, T.D. Märk ${ }^{\text {d,* }}$ \\ a Department of Chemistry, University of Fribourg, Chemin du Musee 9, 1700 Fribourg, Switzerland \\ ${ }^{\mathrm{b}}$ Department of Physics, University of New Hampshire, Durham, NH 03824, USA \\ ${ }^{\mathrm{c}}$ Institute of Physics, Maria Curie-Sklodowska University, 20-031 Lublin, Poland \\ ${ }^{\mathrm{d}}$ Department of Experimental Physics, Comenius University, SK-84248 Bratislava, Slovak Republic \\ e Institute of Ion Physics and Applied Physics, Leopold Franzens Universität, Technikerstrasse 25, A-6020 Innsbruck, Austria
}

\begin{abstract}
We have measured kinetic-energy-release distributions (KERD) for spontaneous dissociation of electronically excited dimer ions of krypton and xenon, formed by electron impact ionization of neutral precursors. The data cannot be reconciled by decay of the strongly bound $\mathrm{II}(1 / 2 \mathrm{u})$ state that successfully explains dissociation of $\mathrm{Ne}_{2}^{+}$and $\mathrm{Ar}_{2}^{+}$. Instead, the KERD is dominated by contributions from the weakly bound $\mathrm{II}(1 / 2 \mathrm{~g})$ state that has so far escaped a convincing experimental characterization. The present data can be utilized to assess the accuracy of $a b$ initio potential energy curves of this state.
\end{abstract}

\section{Introduction}

Rare gas dimer ions are of relevance in a variety of environments. They are ubiquitous in high-pressure plasmas [1]; they are of relevance in VUV rare-gas excimer and ion lasers [2] and plasma display panels [3]. They are crucial to understand the optical properties and dissociation dynamics of rare gas cluster ions [4-10], and they play a role in the localization of charges in rare gas solids [11].

Homonuclear rare gas dimer ions $\mathrm{Rg}_{2}^{+}$other than $\mathrm{He}_{2}^{+}$ possess six low-lying electronic states that converge to the two lowest dissociation limits, $\operatorname{Rg}\left({ }^{1} \mathrm{~S}_{0}\right)+\operatorname{Rg}^{+}\left({ }^{2} \mathrm{P}_{3 / 2}\right)$ and $\operatorname{Rg}\left({ }^{1} \mathrm{~S}_{0}\right)+\operatorname{Rg}^{+}\left({ }^{2} \mathrm{P}_{1 / 2}\right)$. The calculation of their potential energy curves has attracted considerable interest [12-17]. The results of recent ab initio calculations for $\mathrm{Kr}_{2}^{+}$[16] and $\mathrm{Xe}_{2}^{+}$[15] are graphed in Fig. 1. Moreover, a rich body of experimental data has been obtained [18], most recently by high-resolution threshold photoelectron spectroscopy

\footnotetext{
* Corresponding authors. Fax: +16038622998.

E-mail addresses: olof.echt@unh.edu (O. Echt), tilmann.maerk@, uibk.ac.at (T.D. Märk).
}

[4,19-24]. There is good agreement between ab initio calculations and experimental data with one notable exception, the II $(1 / 2 \mathrm{~g})$ state of $\mathrm{Kr}_{2}^{+}$. In early theoretical work $[12,13]$ it was concluded that this state is repulsive. More recent ab initio studies find a dissociation energy $D_{0}=202 \mathrm{~cm}^{-1}$ [16] or $103 \mathrm{~cm}^{-1}$ [17], much smaller than the experimental value of $\approx 600 \mathrm{~cm}^{-1}[20,22]$.

However, the experimental value is problematic. In spite of the high experimental resolution of $<1 \mathrm{~cm}^{-1}$ [22], only one line without any substructure is observed in the photoelectron spectra, slightly above the vibrational progression that arises from transitions to the $\mathrm{II}(1 / 2 \mathrm{u})$ state. The line has been tentatively assigned to a transition from the vibrational ground state of the neutral dimer $\left(\mathrm{X} 0_{\mathrm{g}}^{+}\right)$to the $v^{+}=0$ state of $\mathrm{Kr}_{2}^{+}(\mathrm{II}(1 / 2 \mathrm{~g}))$ [22]. The interpretation implies that the equilibrium distances $R_{\mathrm{e}}$ of $\mathrm{Kr}_{2}^{+}(\mathrm{II}(1 / 2 \mathrm{~g}))$ and the neutral dimer are similar whereas recent ab initio studies find that $R_{\mathrm{e}}(\mathrm{II}(1 / 2 \mathrm{~g}))$ exceeds $R_{\mathrm{e}}\left(\mathrm{X} 0_{\mathrm{g}}^{+}\right)$by $16 \%[16]$ or even $21 \%$ [17] $\left(R_{\mathrm{e}}\right.$ values are indicated in Fig. 1 by vertical arrows).

The situation is not quite as contradictory for $\mathrm{Xe}_{2}^{+}(\mathrm{II}(1 /$ 2g)) where, again, only a single line is observed in 




Fig. 1. Ab initio potential energy (PE) curves for the six lowest electronic states of: (a) $\mathrm{Kr}_{2}^{+}$[16] and (b) $\mathrm{Xe}_{2}^{+}$[15]. Downward arrows indicate transitions discussed in this work and indicate the equilibrium separations $R_{\mathrm{e}}$ in the II(1/2) states. Upward arrows indicate $R_{\mathrm{e}}$ values of the neutral dimers.

photoelectron spectra above the II $(1 / 2 \mathrm{u})$ vibrational progression. According to Rupper et al. [24] the single line is $424.6 \mathrm{~cm}^{-1}$ below the dissociation limit into $\mathrm{Xe}^{+}\left({ }^{2} \mathrm{P}_{1 / 2}\right)$, in good agreement with an ab initio value of $D_{0}=359 \mathrm{~cm}^{-1}$ for the dissociation energy of $\mathrm{Xe}_{2}^{+}(\mathrm{II}(1 / 2 \mathrm{~g})$ [15]. Also, for xenon the calculated equilibrium separation of this state coincides more closely, within $10 \%$, with $R_{\mathrm{e}}\left(X 0_{\mathrm{g}}^{+}\right)$[15]. However, in earlier photoelectron spectra $\mathrm{Lu}$ et al. [19] had identified a single line at considerably higher energy, $842 \mathrm{~cm}^{-1}$.

In summary, the lack of vibrational progressions in the II $(1 / 2 \mathrm{~g})$ state of $\mathrm{Kr}_{2}^{+}$and $\mathrm{Xe}_{2}^{+}$limits the information that can be deduced from spectroscopic data. The experimental value for the dissociation energy of $\mathrm{Kr}_{2}^{+}(\mathrm{II}(1 / 2 \mathrm{~g}))$, in particular, is questionable; it has not even been established with certainty if bound vibrational levels exist for this state [17]. Thus, the accuracy of ab initio calculations for the II $(1 / 2 \mathrm{~g})$ state which are particularly demanding for the heavier rare gas dimer ions because of the large number of electrons and the large spin-orbit splitting cannot be assessed.

In the present work we embark on such an assessment by measuring the kinetic energy released in metastable dissociation of $\mathrm{Kr}_{2}^{+}$and $\mathrm{Xe}_{2}^{+}$. These distributions can be very sensitive to details of the underlying electronic potential energy curves of decaying dimer states [25]. Using this technique we have previously shown [26] that radiative decay of the II $(1 / 2 \mathrm{u})$ state into $\mathrm{I}(1 / 2 \mathrm{~g})$ explains the spontaneous dissociation of $\mathrm{Ar}_{2}^{+}$, while $\mathrm{Ne}_{2}^{+}$also decays by radiationless transition into $\mathrm{I}(3 / 2 \mathrm{u})$. As shown by Yoshii et al. $[4,21]$ the radiative $\mathrm{I}(1 / 2 \mathrm{~g}) \leftarrow \mathrm{II}(1 / 2 \mathrm{u})$ transition is also relevant for spontaneous decay of $\mathrm{Kr}_{2}^{+}$and $\mathrm{Xe}_{2}^{+}$. However, in the current work using a novel three sector field mass spectrometric technique we demonstrate that this transition does not explain the measured kinetic energy release distributions (KERD) for $\mathrm{Kr}_{2}^{+}$and $\mathrm{Xe}_{2}^{+}$. Instead, radiative transitions from the $\mathrm{II}(1 / 2 \mathrm{~g})$ state dominate. $\mathrm{Kr}_{2}^{+}$shows only one transition, into $\mathrm{I}(1 / 2 \mathrm{u})$, while $\mathrm{Xe}_{2}^{+}$shows two transitions, into $I(1 / 2 u)$ and $I(3 / 2 u)$. These data will be used to assess the accuracy of potential energy curves of the II $(1 /$ $2 \mathrm{~g}$ ) state calculated by ab initio methods [15-17] and a semiempirical model [24].

\section{Experimental set-up}

The apparatus consists of a high-resolution double focusing mass spectrometer combined with a second electrostatic analyzer $[27,28]$. Neutral rare gas dimers are produced by expanding either krypton or xenon at about 1 bar through a $20 \mu \mathrm{m}$ nozzle into vacuum; molecules are ionized by electron impact at typically $100 \mathrm{eV}$. The ions are extracted by an electric field and accelerated into the mass spectrometer. They pass through the first field free region, are momentum-analyzed by a magnetic sector field followed by a second field-free region, are energy-selected by a $90^{\circ}$ electric sector field followed by a third field free region, pass through a second electrostatic sector field, and are detected by a channeltron-type electron multiplier.

To study the spontaneous decay of the ions and the kinetic energy released in the reaction, an improved mass analyzed ion kinetic energy (MIKE) technique has been applied [29]. MIKE spectra are recorded by tuning the magnet and first electric sector to transmit the parent ion and scanning the sector field voltage of the second electric sector. In this mode, the mass resolution is sufficient to separate different isotopomers of $\mathrm{Kr}_{2}^{+}$and $\mathrm{Xe}_{2}^{+}$. Stable parent ions of mass $m_{\mathrm{p}}$ pass the second electric sector at the voltage of $U_{\mathrm{p}}=510.6 \mathrm{~V}$; fragment ions $m_{\mathrm{f}}$ produced between the two electric sectors pass at a reduced voltage $U_{\mathrm{p}} m_{\mathrm{f}} / m_{\mathrm{p}}$. The shape of the fragment ion peak reflects, after deconvolution with the parent ion peak, the kinetic energy release distribution (KERD) of the reaction.

\section{Results and discussion}

Fig. 2a shows three MIKE scans of xenon dimer ions with a nominal parent mass of 264, 267 and $272 \mathrm{u}$ respectively. Xenon contains nine naturally occurring isotopes with masses 124, 126, 128, 129, 130, 131, 132, 134 and $136 \mathrm{u}$ with abundances of $0.1 \%, 0.09 \%, 1.91 \%, 26.4 \%$, $4.1 \%, 21.2 \%, 26.9 \%, 10.4 \%$ and $8.9 \%$, respectively. The parent ion at $264 \mathrm{u}$ is most intense; it gives rise to an intense MIKE peak at $255.3 \mathrm{~V}$ (Fig. 2a top). However, it is not suitable for a KERD analysis, because its main contribution $\left({ }^{132} \mathrm{Xe}^{132} \mathrm{Xe}^{+}\right)$is contaminated by ${ }^{130} \mathrm{Xe}^{134} \mathrm{Xe}^{+}$and ${ }^{128} \mathrm{Xe}^{136} \mathrm{Xe}^{+}$. Upon dissociation these contaminants produce fragment ion peaks at $U_{\mathrm{f}}=U_{\mathrm{p}} m_{\mathrm{f}} / m_{\mathrm{p}}=247.6,251.4$, 

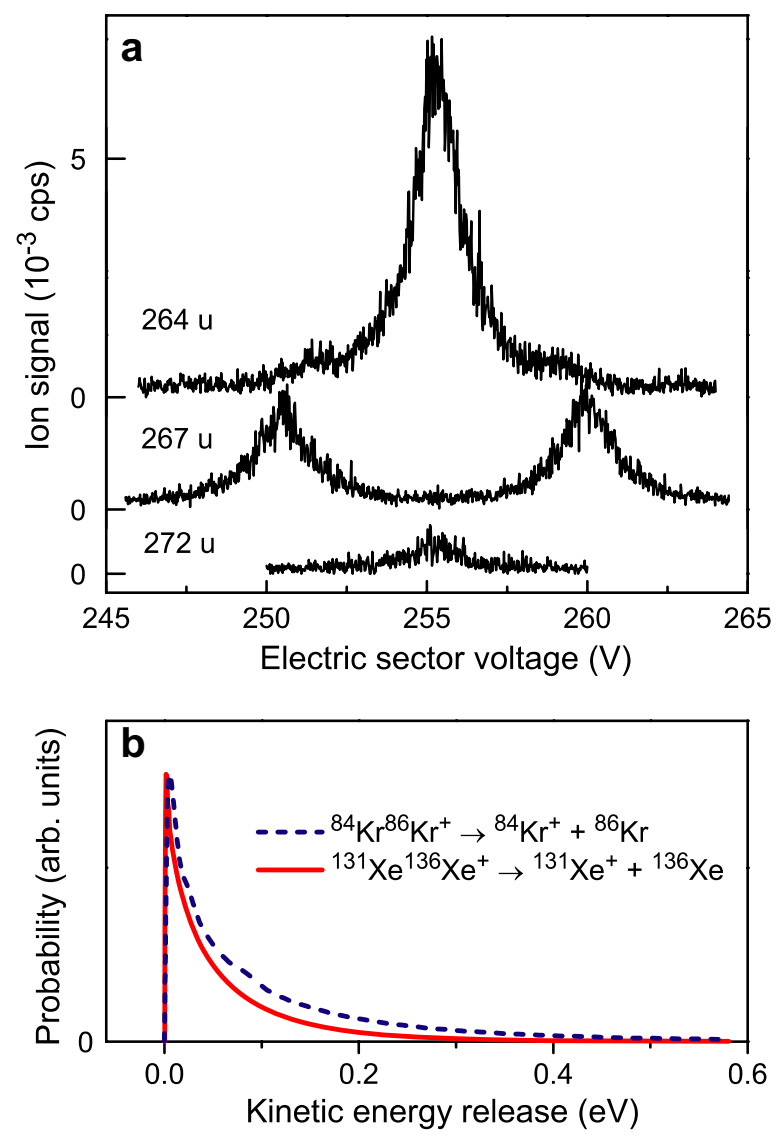

Fig. 2. (a) MIKE spectra for spontaneous dissociation of $\mathrm{Xe}_{2}^{+} \rightarrow \mathrm{Xe}^{+}+$ $\mathrm{Xe}$ for three different parent ion masses. (b) Kinetic energy release distributions (KERD) derived from the MIKE spectrum of $\mathrm{Xe}_{2}^{+}$(mass $267 \mathrm{u})$ and $\mathrm{Kr}_{2}^{+}$(mass $170 \mathrm{u}$ ).

259.2 and $263.0 \mathrm{~V}$, they form barely visible bumps in the wings of the main MIKE peak.

In contrast, the parent ions at 267 and $272 \mathrm{u}$ consist of only one isotopomer each, namely ${ }^{131}{ }^{\mathrm{Xe}^{136}} \mathrm{Xe}^{+}$and ${ }^{136} \mathrm{Xe}^{136} \mathrm{Xe}^{+}$, respectively. The advantage of having only one fragment channel for the ${ }^{136} \mathrm{Xe}^{136} \mathrm{Xe}^{+}$parent is compromised by its very low intensity. Therefore, we choose the mixed dimer ${ }^{131} \mathrm{Xe}^{136} \mathrm{Xe}^{+}$for further analysis. The KERD is obtained by taking the derivative of the MIKE peak, properly deconvoluted with the shape of the parent ion peak, and transforming the sector field voltage to kinetic energies in the center of mass [30]. We have previously run extensive numerical simulations of ion trajectories following reactions with specified KERDs [27]. The study verified that our conversion from MIKE spectra to KERD is accurate and numerically robust, especially for relatively small kinetic energy releases as observed in the present study. Only one side of a MIKE peak is required once the exact center of the peak has been determined. In the present situation we choose the outer wing of the left peak which corresponds to ${ }^{131} \mathrm{Xe}^{+}$fragments. A straightforward estimate shows that the contamination by its twin peak $\left({ }^{136} \mathrm{Xe}^{+}\right.$fragments $)$is no more than a few percent. The KERD thus derived is shown in Fig. $2 b$. The average value of the monotonically decreasing distribution is $33 \mathrm{meV}$.
Yoshii et al. reported an upper limit of $150 \mathrm{meV}$ [21] for the average kinetic energy released in the dissociation of $\mathrm{Xe}_{2}^{+}$following photoexcitation into the $v^{+}=6$ state of $\mathrm{II}(1 / 2 \mathrm{u})$. In contrast, as will be shown below, the main contribution to our experimental signal derives from metastable dissociation of the $\mathrm{II}(1 / 2 \mathrm{~g})$ state. Furthermore, the spectra by Yoshii et al. are dominated by prompt fragments; their experimental technique makes it impossible to unambiguously derive the KERD for metastable reactions. A detailed comparison of our data with their results would therefore be futile.

Krypton has six naturally occurring isotopes; it was analyzed similarly. Among all pure isotopomers, the ion at $170 \mathrm{u}\left({ }^{84} \mathrm{Kr}^{86} \mathrm{Kr}^{+}\right)$is the most abundant one; it produces a split MIKE peak corresponding to the fragment ions ${ }^{84} \mathrm{Kr}^{+}$and ${ }^{86} \mathrm{Kr}^{+}$, respectively. The splitting is large and the contamination of the outer wings of the doublet by their twin peaks is accordingly small. The KERD derived from the MIKE peak (which will be shown later) is displayed in Fig. 2b. The average value of the distribution is $56 \mathrm{meV}$. Yoshii et al. [4,21] reported a value of $<80 \mathrm{meV}$ for dissociation of $\mathrm{Kr}_{2}^{+}\left(\mathrm{II}(1 / 2 \mathrm{u})\left(v^{+}=2\right)\right.$.

For a comparison of experimental data with theory we proceed as follows (see [28] for details)

(1) We calculate the initial population $N_{\mathrm{v}}(t=0)$ of vibronic states of $\mathrm{Kr}_{2}^{+}$and $\mathrm{Xe}_{2}^{+}$at time $t=0$. Only bound states above the dissociation limit into $\operatorname{Rg}\left({ }^{1} \mathrm{~S}_{0}\right)+\operatorname{Rg}^{+}\left({ }^{2} \mathrm{P}_{3 / 2}\right)$ will lead to dissociation into $\mathrm{Rg}^{+}$, i.e. only the $\mathrm{II}(1 / 2 \mathrm{u})$ and $\mathrm{II}(1 / 2 \mathrm{~g})$ states have to be considered (higher states are ignored). For $\mathrm{Kr}_{2}^{+}$we use the ab initio curves by Kalus et al. [16] who used a coupled cluster approach (RHFRCCSD-T) and relativistic effective core pseudo potential, and the curves by Ha et al. [17] obtained from non-relativistic configuration interaction $a b$ initio calculations. For $\mathrm{Xe}_{2}^{+}$we use the ab initio curves by Paidarova and Gadea [15] who used a coupled cluster approach (RHF-RCCSD-T) and relativistic effective core potentials, and PE curves by Rupper et al. [24] derived from spectroscopic data and a global model. Both sets of $\mathrm{PE}$ curves for $\mathrm{Xe}_{2}^{+}$rest on the assumption of the spin-orbit coupling being independent of the interatomic separation. The population $N_{\mathrm{v}}(t=0)$ of vibrational states in the $\mathrm{II}(1 / 2)$ states are given by the Franck-Condon factors from the neutral dimer PE curves [31,32].

(2) In a second step we compute the vibrational population at the time of dissociation $(36.7 \mu \mathrm{s} \leqslant t \leqslant 44.4 \mu \mathrm{s}$ for $\mathrm{Kr}_{2}^{+}$and $46.0 \mu \mathrm{s} \leqslant t \leqslant 55.6 \mu \mathrm{s}$ for $\mathrm{Xe}_{2}^{+}$) from $N_{\mathrm{v}}(t)=N_{\mathrm{v}}(t) \exp \left(-A_{\mathrm{v}} t\right) . \quad A_{\mathrm{v}}$ is the sum of all (bound-bound and bound-free) decay rates from a given vibronic level. Only the $J=0$ rotational quantum numbers are taken into account because the large reduced mass of the heavy rare gas dimer ions leads to a small dependence of the effective potential on $J$. 
(3) For each vibronic level that has a non-negligible population $N_{\mathrm{v}}(t)$ we compute the kinetic energy release distribution, i.e., the coefficients for radiative transitions as a function of the final energy in the continuum.

(4) For each electronic transition we combine the results of (2) and (3).

(5) For a quantitative comparison between experiment and theory we transform the 'predicted' KERD to the laboratory reference system by inverting the procedure described above. A comparison of MIKE spectra is preferred over a comparison of KER distributions because differentiation of noisy experimental data would require heavy smoothing [33]. Furthermore, as apparent from Fig. 2b, the statistical accuracy of the data cannot be assessed from the experimental KERD.

The only free parameter in the model is the temperature of the neutral dimer. We assumed a vibrational dimer temperature of $50 \mathrm{~K}$ for $\mathrm{Kr}_{2}$ and $70 \mathrm{~K}$ for $\mathrm{Xe}_{2}$. These values have been obtained by scaling the temperature of $\mathrm{Ar}_{2}$ measured by Raman scattering [34]; they are consistent with temperatures estimated for larger clusters based on the concept of evaporative cooling [35]. Our results do not significantly depend on variations of the temperature within reasonable limits. Why not? The KERD depends on the initial population of rovibrational levels of the ion and the emission rates of those levels; the second of these factors is much more decisive than the first. For example, further below we will show that transitions from the II $(1 / 2 \mathrm{u})$ state are dominated by transitions from the highest bound vibrational level; the population of lower vibrational levels matters little. A detailed discussion of this effect on the decay of $\mathrm{Ar}_{2}^{+}$has been presented elsewhere [28].

The KERD predicted for $\mathrm{Xe}_{2}^{+}$is plotted in Fig. 3. All three dipole-allowed transitions from the two electronic states that correlate with the $\left.\mathrm{Xe}^{1}{ }^{1} \mathrm{~S}_{0}\right)+\mathrm{Xe}^{+}\left({ }^{2} \mathrm{P}_{1 / 2}\right)$ dissociation limit are active on the time scale of our experiment. The corresponding three contributions to the KERD, calculated from the ab initio PE curves [15], are plotted separately in Fig. $3 \mathrm{a}$ and those calculated from the semiempirical PE curves [24] are plotted in Fig. 3b. A dominant contribution to the total KERD comes from the $\mathrm{I}(1 /$ $2 \mathrm{u}) \leftarrow \mathrm{II}(1 / 2 \mathrm{~g})$ transition and a weaker one from the perpendicular $\mathrm{I}(3 / 2 \mathrm{u}) \leftarrow \mathrm{II}(1 / 2 \mathrm{~g})$ transition. The final states are bound at internuclear distances of the II(1/2g) state. Hence the nuclear wave functions of the final continuum states have highest amplitudes at energies just above the asymptotic limit, and the predicted KERD decreases essentially monotonically with increasing energy.

A third contribution is predicted to come from transitions of $\mathrm{II}(1 / 2 \mathrm{u})$ into the strongly repulsive part of $\mathrm{I}(1 /$ $2 \mathrm{~g}$ ); they give rise to a high-energy component in the predicted KERD with a most likely value of $0.35 \mathrm{eV}$ for the $\mathrm{ab}$ initio PE curves, and $0.22 \mathrm{eV}$ for the semiempirical curves. Transitions from the highest vibrational state of
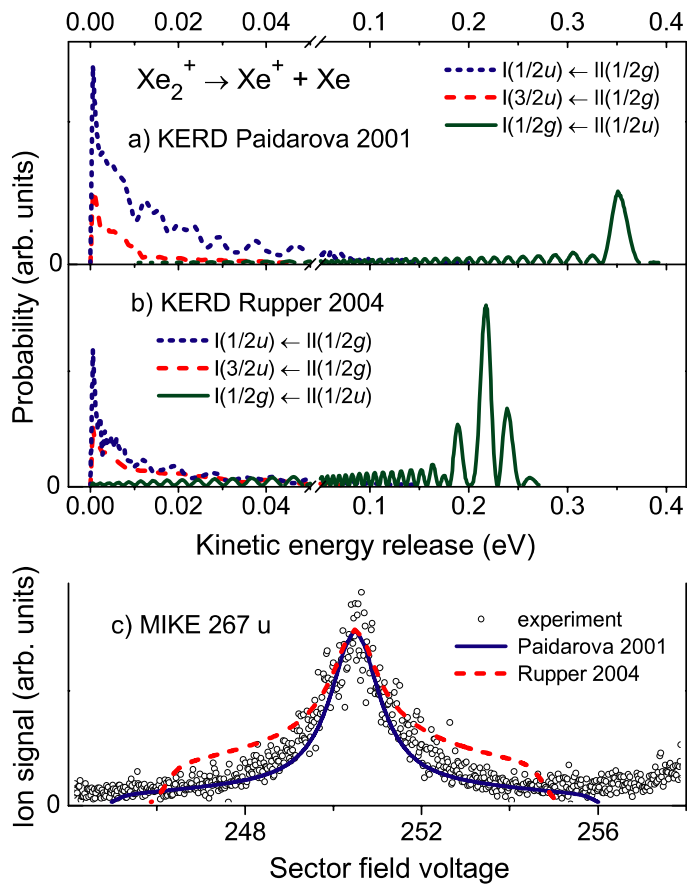

Fig. 3. Kinetic energy release distributions for $\mathrm{Xe}_{2}^{+} \rightarrow \mathrm{Xe}^{+}+\mathrm{Xe}$ calculated for three electronic transitions from ab initio PE curves [15] (panel a) and from the semiempirical PE curves [24] (panel b). For greater clarity, the energy scale is changed at $0.05 \mathrm{eV}$. (c) Comparison of the experimental MIKE spectrum (open dots) with the summed and transformed theoretical KERDs.

$\mathrm{II}(1 / 2 \mathrm{u})$ dominate, we find $v_{\max }=62$ for the ab initio PE curves [15]. However, the highest value of $v$ that we can take into account is limited by numerical instabilities. A change to $v_{\max }=61$ would have a negligible effect on the computed KERD whereas the calculated weight $N_{\mathrm{v}}(t)$ would change by a factor two. Hence, we cannot reliably predict the relative contribution of transitions from the $\mathrm{II}(1 / 2 \mathrm{u})$ state versus those from the II $(1 / 2 \mathrm{~g})$ state.

In the MIKE spectrum (Fig. 3c) the predicted highenergy component would be visible as a wide, roughly rectangular contribution. The semiempirical PE curves predict a step at $\approx 246 \mathrm{~V}$ corresponding to a predicted KER of $0.22 \mathrm{eV}$, whereas the ab initio curves predict a less pronounced step at $\approx 245 \mathrm{~V}(\mathrm{KER}=0.35 \mathrm{eV})$. There is no evidence for such a step in the experimental data which are already explained quite well by the two low-energy components. Thus, on the time scale of our experiment, the $\mathrm{I}(1 /$ $2 \mathrm{~g}) \leftarrow \mathrm{II}(1 / 2 \mathrm{u})$ transition is overwhelmed by the $\mathrm{I}(1 /$ $2 \mathrm{u}) \leftarrow \mathrm{II}(1 / 2 \mathrm{~g})$ and $\mathrm{I}(3 / 2 \mathrm{u}) \leftarrow \mathrm{II}(1 / 2 \mathrm{~g})$ transitions.

We now turn to a discussion of krypton. The experimental MIKE peak of the ${ }^{84} \mathrm{Kr}^{+}$fragment from ${ }^{84} \mathrm{Kr}^{86} \mathrm{Kr}^{+}$is displayed in Fig. 4c. It is similar in shape to the one observed for xenon but wider, reflecting the larger average KER ( $56 \mathrm{meV}$ for krypton versus $33 \mathrm{meV}$ for xenon). Only two electronic transitions are calculated to be significant, namely $\mathrm{I}(1 / 2 \mathrm{u}) \leftarrow \mathrm{II}(1 / 2 \mathrm{~g})$ and $\mathrm{I}(1 / 2 \mathrm{~g}) \leftarrow \mathrm{II}(1 / 2 \mathrm{u})$. The perpendicular $\mathrm{I}(3 / 2 \mathrm{u}) \leftarrow \mathrm{II}(1 / 2 \mathrm{~g})$ transition is four orders of magnitude weaker than $\mathrm{I}(1 / 2 \mathrm{u}) \leftarrow \mathrm{II}(1 / 2 \mathrm{~g})$. As before, the $\mathrm{I}(1 / 2 \mathrm{u}) \leftarrow \mathrm{II}(1 / 2 \mathrm{~g})$ transition results in a monotonically 

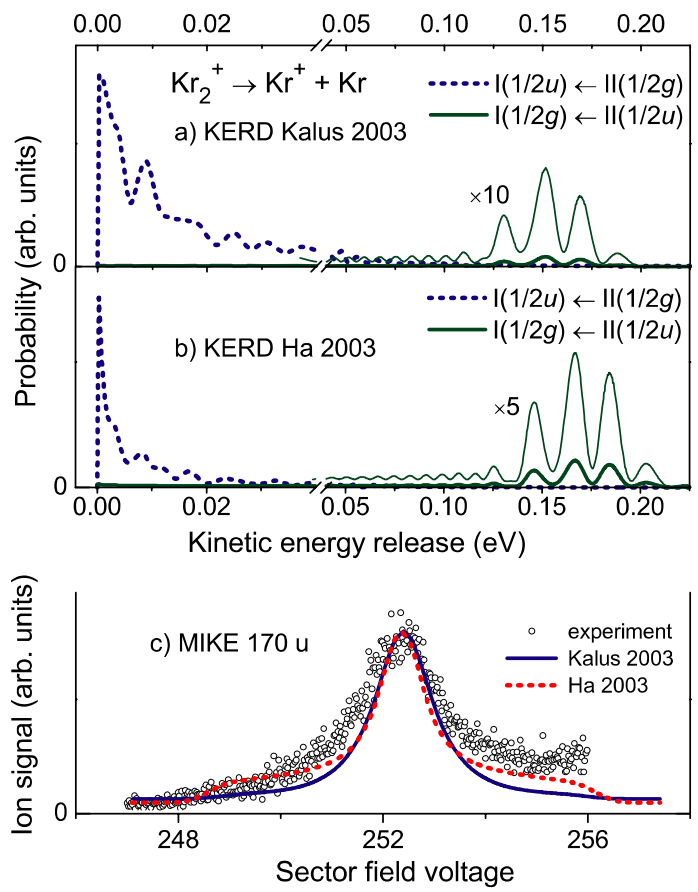

Fig. 4. KERD for $\mathrm{Kr}_{2}^{+} \rightarrow \mathrm{Kr}^{+}+\mathrm{Kr}$ calculated for two electronic transitions from ab initio PE curves by Kalus et al. [16] (panel a) and by Ha et al. [17] (panel b). For greater clarity, the energy scale is changed at $0.04 \mathrm{eV}$, and the high-energy component is amplified by a factor 10 and 5 , respectively. (c) Comparison of the experimental MIKE spectrum (open dots) with the summed and transformed theoretical KERDs.

decreasing KERD whereas the transition from II $(1 / 2 \mathrm{u})$ into the repulsive part of $\mathrm{I}(1 / 2 \mathrm{~g})$ produces a KERD that peaks around $0.15 \mathrm{eV}$; this transition is relatively more intense for the PE curves by $\mathrm{Ha}$ et al. [17] than for the PE curves by Kalus et al. [16], see Fig. 4a,b. This highenergy component is responsible for the step in the predicted MIKE spectrum near $249 \mathrm{~V}$, see Fig. 4c. There is no hint of such a step in the experimental data, i.e. the transition does not significantly contribute (recall that the calculated relative weights of the two transitions carry a large uncertainty).

The $\mathrm{I}(1 / 2 \mathrm{u}) \leftarrow \mathrm{II}(1 / 2 \mathrm{~g})$ transition produces a MIKE peak whose shape matches the experimental data. However, the predicted curves are narrower than the measured one. The discrepancy is more pronounced for the PE curves by Ha et al. [17]. This can be seen in the low-energy part of the KERDs (Fig. 4a,b). When transformed to the laboratory reference system (Fig. 4c), a narrower low-energy component corresponds to a 'predicted' MIKE peak that is narrow near its peak.

\section{Conclusions}

In conclusion, we have measured the distributions of kinetic energy released in the metastable reaction $\mathrm{Rg}_{2}^{+} \rightarrow \mathrm{Rg}^{+}+\mathrm{Rg}$ for carefully chosen isotopomers of $\mathrm{Kr}_{2}^{+}$and $\mathrm{Xe}_{2}^{+}$. At the same time we have 'predicted' these distributions based on published potential energy curves for $\mathrm{Kr}_{2}^{+}$and $\mathrm{Xe}_{2}^{+}$. Radiative transitions from the $\mathrm{II}(1 / 2 \mathrm{u})$ and the $\mathrm{II}(1 / 2 \mathrm{~g})$ state are expected to contribute. Comparison with the experimental data shows that transitions from the $\mathrm{II}(1 / 2 \mathrm{~g})$ state dominate, in contrast to decay of $\mathrm{Ne}_{2}^{+}$and $\mathrm{Ar}_{2}^{+}$where the situation is reverse. The data thus establish, beyond any doubt, that the $\mathrm{Kr}_{2}^{+}(\mathrm{II}(1 / 2 \mathrm{~g}))$ state supports bound vibrational levels. However, the agreement of the 'predicted' distributions with experimental data is, especially for krypton, only modest, suggesting that the calculated $\mathrm{II}(1 / 2 \mathrm{~g})$ potential energy curves are still lacking in accuracy.

\section{Acknowledgements}

The authors thank I. Paidarova and F. Merkt for providing PE curves of rare gas dimer ions, and F. Merkt and J.N. Harvey for suggestions and fruitful discussions. This work was partly supported by the FWF, Wien, and by the EU Commission, Brussels.

\section{References}

[1] J. Samson, D.L. Ederer, Vacuum Ultraviolet Spectroscopy, Academic Press, San Diego, 2000.

[2] J.J. Ewing, IEEE J. Sel. Top. Quantum Electron. 6 (2000) 1061.

[3] H.S. Uhm, E.H. Choi, K.B. Jung, Phys. Plasmas 12 (2005) 033507.

[4] H. Yoshii, K. Tsukamoto, T. Hayaishi, T. Aoto, K. Ito, Y. Morioka, J. Chem. Phys. 123 (2005) 184303.

[5] B. von Issendorff, A. Hofmann, H. Haberland, J. Chem. Phys. 111 (1999) 2513.

[6] A.B. Jones, P.R. Jukes, A.J. Stace, J. Chem. Phys. 111 (1999) 959.

[7] Z.Y. Chen, C.D. Cogley, J.H. Hendricks, B.D. May, A.W. Castleman, J. Chem. Phys. 93 (1990) 3215.

[8] M.J. DeLuca, M.A. Johnson, Chem. Phys. Lett. 162 (1989) 445.

[9] T.D. Märk, O. Echt, in: H. Haberland (Ed.), Clusters of Atoms and Molecules II, vol. 56, Springer-Verlag, Berlin, 1994, p. 154.

[10] C.P. Malone, W. Kedzierski, J.W. McConkey, J. Phys. B 36 (2003) 3607.

[11] N. Schwentner, E.E. Koch, J. Jortner, Electronic Excitations in Condensed Rare Gases, Springer, Heidelberg, 1985.

[12] W.R. Wadt, J. Chem. Phys. 68 (1978) 402.

[13] H.H. Michels, R.H. Hobbs, L.A. Wright, J. Chem. Phys. 69 (1978) 5151.

[14] M. Daskalopoulou, H.-U. Böhmer, S.D. Peyerimhoff, Z. Phys. D 15 (1990) 161.

[15] I. Paidarova, F.X. Gadea, Chem. Phys. 274 (2001) 1.

[16] R. Kalus, I. Paidarova, D. Hrivnak, P. Paska, F.X. Gadea, Chem. Phys. 294 (2003) 141.

[17] T.K. Ha, P. Rupper, A. Wüest, F. Merkt, Mol. Phys. 101 (2003) 827.

[18] M.L. Ginter, J.G. Eden, Can. J. Phys. 82 (2004) 762.

[19] Y. Lu et al., J. Chem. Phys. 102 (1995) 1553.

[20] R. Hall et al., J. Phys. B: At. Mol. Opt. Phys 28 (1995) 2435.

[21] H. Yoshii, T. Hayaishi, T. Onuma, T. Aoto, Y. Morioka, K. Ito, J. Chem. Phys. 117 (2002) 1517.

[22] R. Signorell, U. Hollenstein, F. Merkt, J. Chem. Phys. 114 (2001) 9840.

[23] A. Wüest, F. Merkt, Mol. Phys. 103 (2005) 1285.

[24] P. Rupper, O. Zehnder, F. Merkt, J. Chem. Phys. 121 (2004) 8279.

[25] N. Moiseyev, R. Santra, J. Zobeley, L.S. Cederbaum, J. Chem. Phys. 114 (2001) 7351.

[26] J. Fedor et al., Phys. Rev. Lett. 91 (2003) 133401.

[27] S. Matt et al., Int. J. Mass Spectrom. 187 (1999) 813.

[28] J. Fedor, K. Gluch, R. Parajuli, S. Matt-Leubner, O. Echt, P. Scheier, T.D. Märk, J. Chem. Phys. 121 (2004) 7253. 
[29] K. Gluch et al., J. Chem. Phys. 120 (2004) 2686.

[30] M.F. Jarrold, J. Illies, N.J. Kirchner, W. Wagner-Redeker, M.T. Bowers, M.L. Mandich, J.L. Beauchamp, J. Phys. Chem. 87 (1983) 2213.

[31] R.A. Aziz, M.J. Slaman, Mol. Phys. 58 (1986) 679.
[32] R.A. Aziz, M.J. Slaman, Mol. Phys. 57 (1986) 825.

[33] K. Gluch, S. Matt-Leubner, O. Echt, R. Deng, J.U. Andersen, P. Scheier, T.D. Märk, Chem. Phys. Lett. 385 (2004) 449.

[34] H.P. Godfried, I.F. Silvera, Phys. Rev. A 27 (1982) 3008.

[35] C.E. Klots, Nature 327 (1987) 222. 